\title{
In vivo genome-wide profiling reveals a tissue-specific role for 5-formylcytosine
}

Mario lurlaro ${ }^{1+}$ (D), Gordon R. Mclnroy ${ }^{2+}{ }^{\text {, Heather E. Burgess }}{ }^{1}$, Wendy Dean ${ }^{1}$, Eun-Ang Raiber ${ }^{3}$, Martin Bachman ${ }^{2,3,4}$, Dario Beraldi ${ }^{2}$, Shankar Balasubramanian ${ }^{2,3,5^{*}}$ and Wolf Reik ${ }^{1,6,7^{*}}$

\begin{abstract}
Background: Genome-wide methylation of cytosine can be modulated in the presence of TET and thymine DNA glycosylase (TDG) enzymes. TET is able to oxidise 5-methylcytosine ( $5 \mathrm{mC})$ to 5 -hydroxymethylcytosine (5hmC), 5-formylcytosine (5fC) and 5-carboxylcytosine (5caC). TDG can excise the oxidative products $5 f C$ and $5 \mathrm{caC}$, initiating base excision repair. These modified bases are stable and detectable in the genome, suggesting that they could have epigenetic functions in their own right. However, functional investigation of the genome-wide distribution of $5 f C$ has been restricted to cell culture-based systems, while its in vivo profile remains unknown.

Results: Here, we describe the first analysis of the in vivo genome-wide profile of $5 \mathrm{fC}$ across a range of tissues from both wild-type and Tdg-deficient E11.5 mouse embryos. Changes in the formylation profile of cytosine upon depletion of TDG suggest TET/TDG-mediated active demethylation occurs preferentially at intron-exon boundaries and reveals a major role for TDG in shaping $5 f C$ distribution at $\mathrm{CpG}$ islands. Moreover, we find that active enhancer regions specifically exhibit high levels of $5 f C$, resulting in characteristic tissue-diagnostic patterns, which suggest a role in embryonic development.

Conclusions: The tissue-specific distribution of $5 \mathrm{fC}$ can be regulated by the collective contribution of TET-mediated oxidation and excision by TDG. The in vivo profile of $5 f C$ during embryonic development resembles that of embryonic stem cells, sharing key features including enrichment of $5 \mathrm{fC}$ in enhancer and intragenic regions. Additionally, by investigating mouse embryo $5 \mathrm{fC}$ profiles in a tissue-specific manner, we identify targeted enrichment at active enhancers involved in tissue development.
\end{abstract}

\section{Background}

Active DNA demethylation in mammals can be achieved via iterative oxidation of 5-methylcytosine $(5 \mathrm{mC})$ by one or more of the TET enzymes to generate 5 hydroxymethylcytosine $(5 \mathrm{hmC})$, 5-formylcytosine $(5 \mathrm{fC})$ and 5-carboxylcytosine $(5 \mathrm{caC})$ [1-5]. $5 \mathrm{fC}$ and $5 \mathrm{caC}$ are recognised and excised by the thymine DNA glycosylase (TDG) enzyme, initiating base excision repair (BER). Alternatively, $5 \mathrm{fC}$ may be lost passively during DNA replication $[5,6]$. Following the initial identification of $5 \mathrm{fC}$ as an oxidative product of $5 \mathrm{mC}, 5 \mathrm{fC}$ has been detected in a variety of tissues and cell systems, albeit at relatively low levels compared to $5 \mathrm{mC}$

\footnotetext{
*Correspondence: sb10031@cam.ac.uk; wolf.reik@babraham.ac.uk ${ }^{\dagger}$ Equal contributors

${ }^{2}$ Department of Chemistry, University of Cambridge, Lensfield Road, Cambridge CB2 1EW, UK

${ }^{1}$ The Babraham Institute, Epigenetics Programme, Cambridge CB22 3AT, UK Full list of author information is available at the end of the article
}

and $5 \mathrm{hmC}[4,7]$. Recently we demonstrated that $5 \mathrm{fC}$ is not only detectable but also stable in vivo [8], lending credence to a biological role for $5 \mathrm{fC}$ beyond that of a demethylation intermediate. Indeed, several proteins have been found to specifically bind $5 \mathrm{fC}$ in mouse embryonic stem cells (mESCs) and neural precursor cells (NPCs) with putative roles in transcription, chromatin regulation and DNA repair $[9,10]$. The mechanisms by which $5 \mathrm{fC}$ affects these processes are not known, but it has recently been proposed that changes in the physical properties of the DNA double-stranded helix may be involved [11].

In the past three years, a number of different methods have been described for the mapping of $5 \mathrm{fC}$ in the genome of mESCs. These methods fall broadly into two categories: (1) affinity enrichment methods relying on antibody or chemical pull-downs [12-14]; and (2) base-resolution methods based on modifications to the classical bisulphite 
sequencing (BS-seq) technique [13, 15-17]. Recently two bisulphite-free base-resolution methods have been described that employ either the change in base-pairing properties ( $\mathrm{C}$ to $\mathrm{T}$ ) upon chemical modification [18] or the use of a specific restriction enzyme followed by an enrichment step [19]. Despite the methodological differences between these techniques, a consistent picture of the distribution of $5 \mathrm{fC}$ in mESCs is emerging. $5 \mathrm{fC}$ is enriched at enhancers, repetitive elements and exons, but how this relates to its distribution in tissues of a whole organism is currently unexplored. Considering the dynamic nature of the modification and its enrichment at regulatory regions, this becomes extremely relevant especially in the context of development, where epigenetic modifications are subject to changes.

Here we present the first in vivo genome-wide profile of $5 \mathrm{fC}$, in tissues of the early mouse embryo. In particular, we describe the distribution of $5 \mathrm{fC}$ in the hindbrain, heart, liver and carcass of embryonic day (E) 11.5 wildtype (WT) and $T d g$ null embryos. The use of $T d g$ null embryos enables the identification of sites marked by active demethylation driven by TET/TDG activity in these tissues, highlighting a potential role for $5 \mathrm{fC}$ in tissue development and differentiation.

\section{Results and discussion}

\section{Genomic levels of $5 \mathrm{fC}$ are heterogeneous in} mid-gestation staged embryos

We quantified genomic levels of $5 \mathrm{fC}$ from DNA extracted from whole embryos at various developmental stages between E9.5 and E12.5 by liquid chromatography-mass spectrometry (LC/MS) (Fig. 1a). We found that in mid-gestational embryos, $5 \mathrm{fC}$ is present at levels comparable to mouse ES cells, reaching a maximum at E11.5-E12.5. Mice lacking the TDG enzyme show substantially higher levels of $5 \mathrm{fC}$ (approximately a sevenfold increase) in accordance with its ability to excise $5 \mathrm{fC}$
(Additional file 1: Figure S1A) [6]. As previously reported [20, 21], Tdg null mice displayed embryonic lethality after E11.5 with a distinct phenotype characterised by haemorrhaging. We therefore decided to restrict our focus to E11.5 embryos for further analysis. We previously quantified genomic $5 \mathrm{fC}$ levels in dissected tissues from WT E11.5 mouse embryos by LC/MS [8]. Quantification of $5 \mathrm{fC}$ levels in the corresponding tissues dissected from Tdg null (TDG null) embryos (Fig. 1b and Additional file 1: Figure S1B) showed that levels of $5 \mathrm{fC}$ in the absence of $T d g$ are heterogeneous. Thus, the highest levels were detected in the carcass and in the hindbrain, where $5 \mathrm{fC}$ was approximately twofold higher than the concentration found in the liver, the tissue with the lowest $5 \mathrm{fC}$ levels in both WT and Tdg null embryos. Notably, this heterogeneity shows no clear correlation with the expression levels of TET and TDG enzymes measured by qPCR in WT tissues (Additional file 1: Figure S1C).

\section{Genome-wide profiling of $5 \mathrm{fC}$ with a traceless chemical probe}

In order to better understand the biological role of $5 \mathrm{fC}$ during embryonic development we generated genomewide profiles for $5 \mathrm{fC}$ in dissected tissues from E11.5 WT and $T d g$ null embryos. To this end, we developed an optimised protocol based on a previously reported 5fC-pull-down method [12], additionally employing a cleavable aldehyde-reactive biotinylation probe as previously described [22] (Additional file 1: Figure S2A). The hydroxylamine moiety exploits the unique reactivity of $5 \mathrm{fC}$ among the other DNA bases to install a biotin tag, enabling subsequent enrichment with streptavidin coated magnetic beads. The presence of a chemical cleavage site (Additional file 1: Figure S2B) enables removal of the biotin tag following enrichment, which alleviates PCR biases introduced by polymerase stalling at modified sites [22]. This new probe and methodology

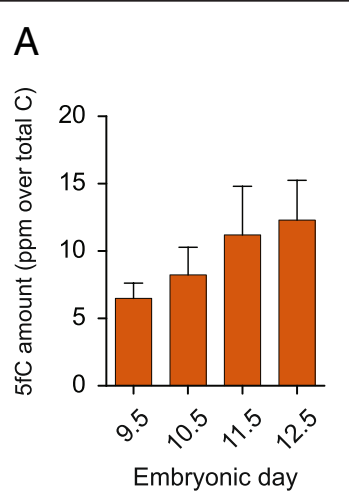

B

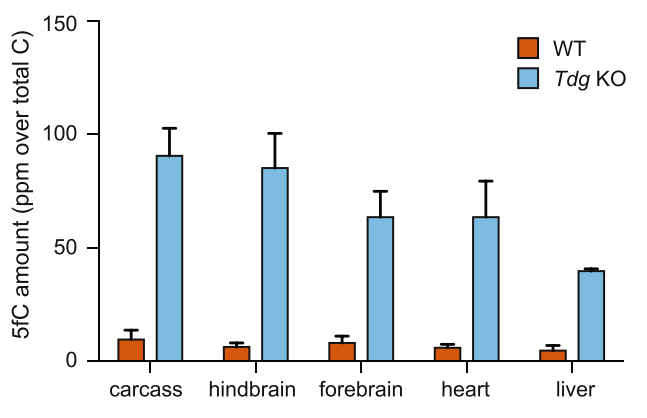

Fig. 1 Genomic $5 f C$ is heterogeneous in early embryos. a LC/MS quantification of genomic $5 f C$ levels in mid-gestation embryos. Displayed is the average of at least three biological replicates with standard deviation. Results are expressed as ppm (part per million over total of cytosines). b LC/MS quantification of genomic 5fC levels in dissected tissues from WT (brown) and Tdg knockout (light blue) E11.5 embryos. Displayed is the average of three biological replicates with standard deviation. Results are expressed as ppm (part per million over total of cytosines) 
resulted in substantially increased enrichment efficiencies over 30 -fold higher than the previously reported method. Through simultaneously obtaining an enhanced signal/noise ratio and increasing resolution, we were able to perform sensitive and robust profiling of $5 \mathrm{fC}$ from relatively small amounts of embryonic-tissuederived genomic DNA (Additional file 1: Figure S2C). We used this capture method to systematically perform genome-wide profiling of $5 \mathrm{fC}$ in the hindbrain, heart, liver and carcass of E11.5 embryos. At least two biological replicates were performed for each tissue and WT and Tdg null samples were paired from the same litter to account for litter-induced variability. A full list of replicates is available in Additional file 1: Figure S3A. In accordance with the data obtained by LC/MS, $T d g$ null embryos show substantially higher levels of $5 \mathrm{fC}$ in all tissues analysed, reflected in the higher signal and increased number of $5 \mathrm{fC}$ peaks in all Tdg null samples compared to their WT counterparts. Biological replicates for the same tissues from different embryos showed an extremely high degree of similarity, suggesting a tight regulation of TET-driven formation and TDG-mediated excision of $5 \mathrm{fC}$ during development. Unbiased hierarchical clustering of all individual replicates accurately separates all samples derived from WT and $T d g$ null embryos, while simultaneously pairing replicates from the same tissues (Additional file 1: Figure S3B). One replicate for the $T d g$ null liver was of poor quality and therefore was excluded from the following analyses.

We then investigated how $5 \mathrm{fC}$ was distributed among functional features within the genome. To this end, $5 \mathrm{fC}$ peaks were initially called independently in each pulldown and enrichment of peaks over genomic features was calculated using the Genomic Association Tester (GAT) [23]. 5fC peaks were found to be most enriched in exons, 5' UTRs and 3' UTRs, while showing lesser enrichment in introns and gene bodies. Moreover, $5 \mathrm{fC}$ was depleted in the intergenic regions of all tissues analysed (Fig. 2a, b and Additional file 1: Figure S4). Although higher levels of $5 \mathrm{fC}$ in $T d g \mathrm{KO}$ tissues result in a higher number of $5 \mathrm{fC}$ peaks called, the distribution of $5 \mathrm{fC}$ peaks over most genomic features was remarkably similar between WT and knockout samples, suggesting that in these regions TDG controls the level of $5 \mathrm{fC}$ without changing its pattern (Fig. 2a, b and Additional file 1: Figure S4). The only exception to this pattern was found in CpG islands (CGIs), where $5 \mathrm{fC}$ peaks were enriched in all WT tissues (with the exception of the carcass), but depleted in the same tissues from embryos lacking $T d g$. It is therefore possible that TDG may be required for TET-mediated oxidation at CpG islands, as suggested by the physical interaction between TET1 and TDG [24], or that excision of $5 \mathrm{fC}$ by TDG is less efficient at these sites. Looking at the distribution across CGIs in more detail, we observed that in $T d g$ null embryos $5 \mathrm{fC}$ is absent from CGIs but enriched at flanking regions, often referred to as 'CGI shores' (Fig. 2c, d and Additional file 1: Figure S5). This clearly indicates a distinctive role for TDG in shaping $5 \mathrm{fC}$ distribution over CpG islands that is conserved across all embryonic tissues. This finding is particularly relevant since aberrant methylation of CGIs has been found in $T d g$ null mice and was suggested as a possible cause of the lethal phenotype [20, 21]. Misregulation of $5 \mathrm{fC}$ may be fundamentally involved in the aberrant methylation described in $T d g$ null embryos, which was previously undetectable owing to the limitation of the methylation analysis technique employed-bisulphite sequencing-to distinguish $5 \mathrm{fC}$ from cytosine [20, 21].

Finally, we observed a substantial enrichment for $5 \mathrm{fC}$ over exon/intron boundaries in both WT and knockout tissues (Fig. 2e, f and Additional file 1: Figure S6). This has also been observed for $5 \mathrm{hmC}$ and $5 \mathrm{fC}$ in embryonic stem cells and suggests a role for oxidised cytosine derivatives in regulating splicing $[14,25]$. The presence of $5 \mathrm{fC}$ at these sites is particularly interesting, as $5 \mathrm{fC}$ has been found to reduce the rate and the fidelity of transcription by RNA polymerase II [26].

\section{$5 \mathrm{fC}$ is enriched at active enhancers in the mouse embryo}

It has been reported that the genomic distribution of $5 \mathrm{fC}$ in $\mathrm{mESCs}$ shows a significant enrichment at distal regulatory regions, mostly enhancers with high levels of mono-methylation of lysine 4 on histone H3 (H3K4me1) but low or absent acetylation of lysine 27 (H3K27ac) [13]. As they are marked by the active H3K4me1, but are still in a repressed deacetylated state, these enhancers are often referred to as 'poised'. In order to investigate the distribution of $5 \mathrm{fC}$ in relation to functional enhancers in vivo, we analysed the $5 \mathrm{fC}$ distribution in E11.5 tissues in relation to available datasets for known histone marks generated in mouse embryos by the ENCODE consortium [27] (Additional file 2: Table S1). We found that in all tissues analysed $5 \mathrm{fC}$ was indeed enriched over distal regulatory regions marked by H3K4me1. The screenshot in Fig. 3a taken from the genome browser shows an example of this striking association. However, in contrast to $\mathrm{mESCs}, 5 \mathrm{fC}$ in embryonic tissues is predominantly enriched over 'active' enhancers marked by H3K4me1 and H3K27ac (Fig. 3b and Additional file 1: Figure S7). We found this to be true in all tissues analysed, regardless of the presence or absence of TDG (although 5fC levels are higher in Tdg null embryos, relative peak enrichment is not). Notably, $5 \mathrm{fC}$ displays a relatively small enrichment over regions marked by tri-methylation of histone $\mathrm{H} 3$ lysine 4 (H3K4me3), a modification associated with promoter regions of active 


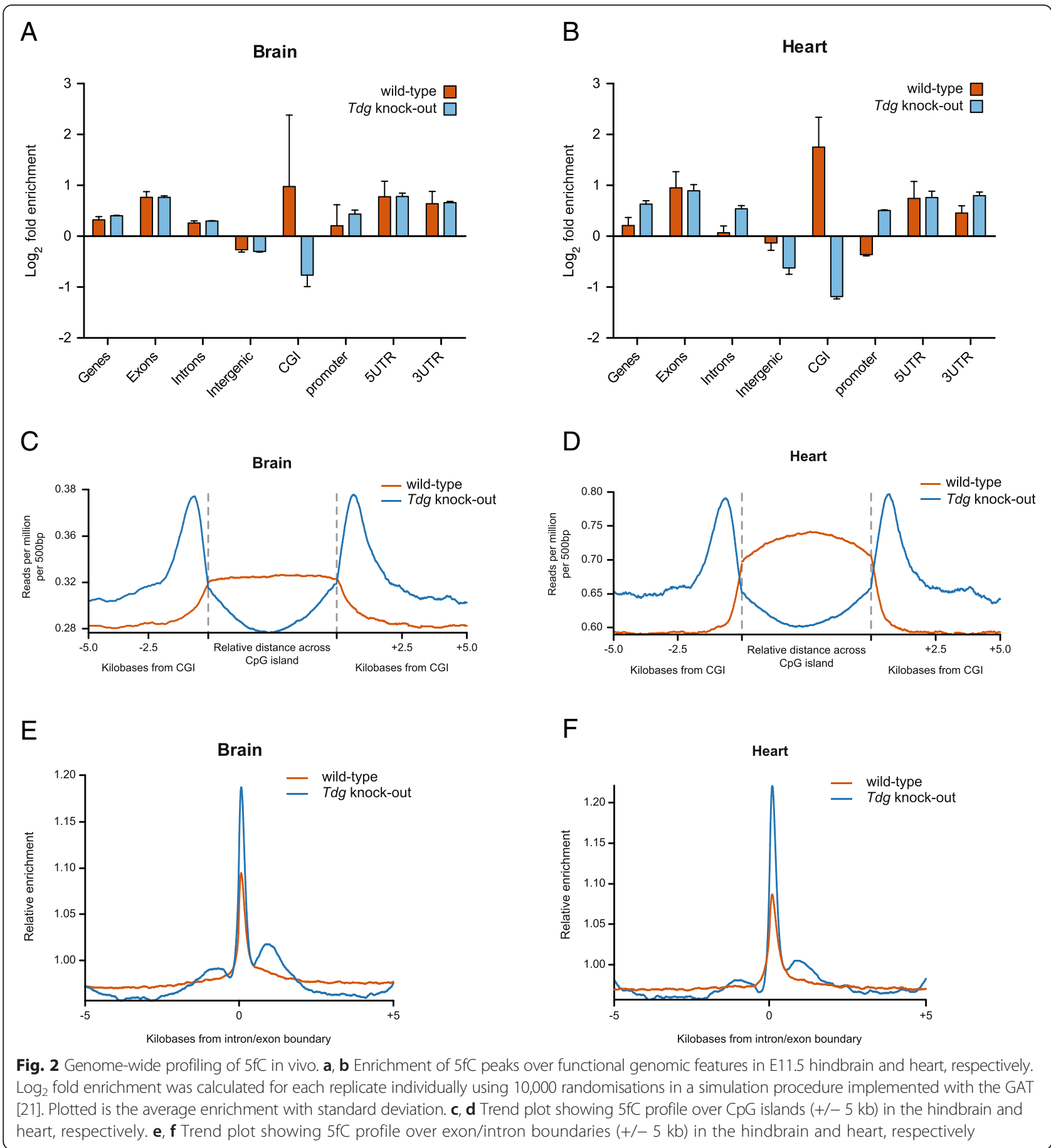

genes and previously reported to show a positive correlation with $5 \mathrm{fC}$ in mESCs (Fig. 3b) [12, 28].

We next asked whether $5 \mathrm{fC}$ enrichment was specific for regions marked by activating histone modifications. To this end, we analysed the distribution of $5 \mathrm{fC}$ peaks in the hindbrain at regions marked by tri-methylation of histone H3 lysine 36 (H3K36me3), which normally labels transcriptional elongation and hence gene bodies, or by tri-methylation of lysine 27 (H3K27me3), a repressive mark deposited by the PRC2 complex. Notably, 5fC shows very little enrichment over these regions (Fig. 3c). Taken together, these data show a strong and specific enrichment of $5 \mathrm{fC}$ at active enhancer regions during embryonic development. This constitutes a significant difference in the profile of $5 \mathrm{fC}$ between mESCs and embryonic tissues and could reflect a different need for dynamic regulation in developing embryos compared to the stem cell state. 
A

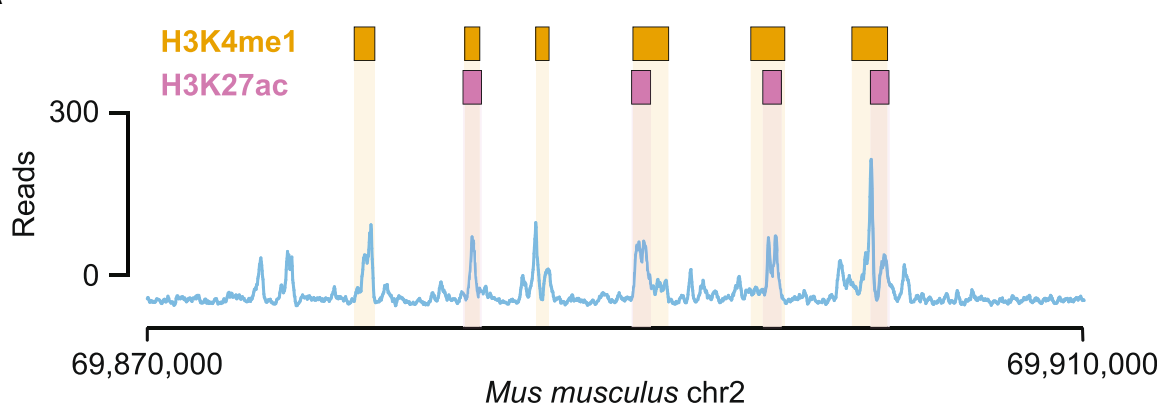

B

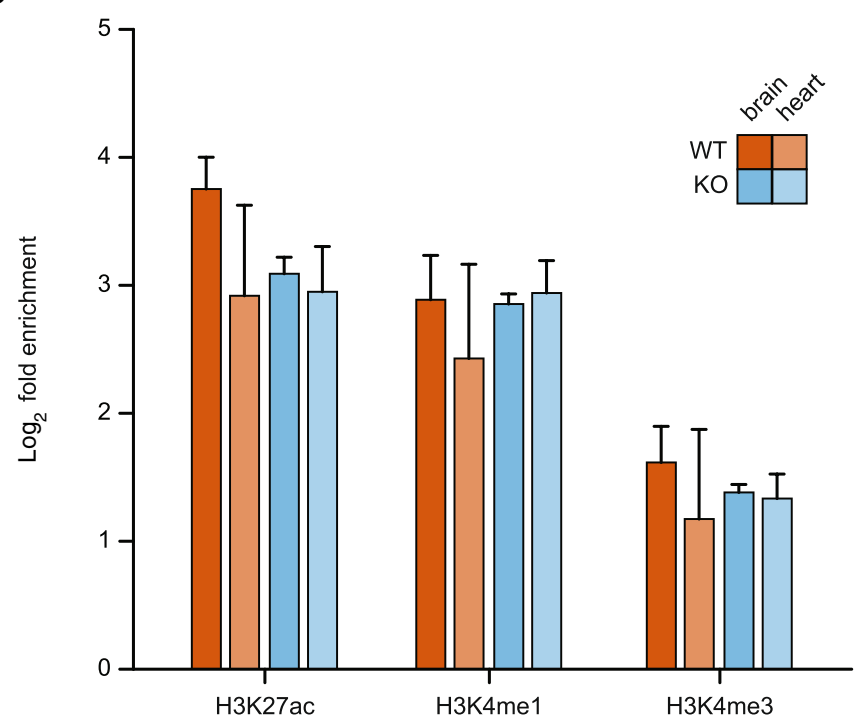

C

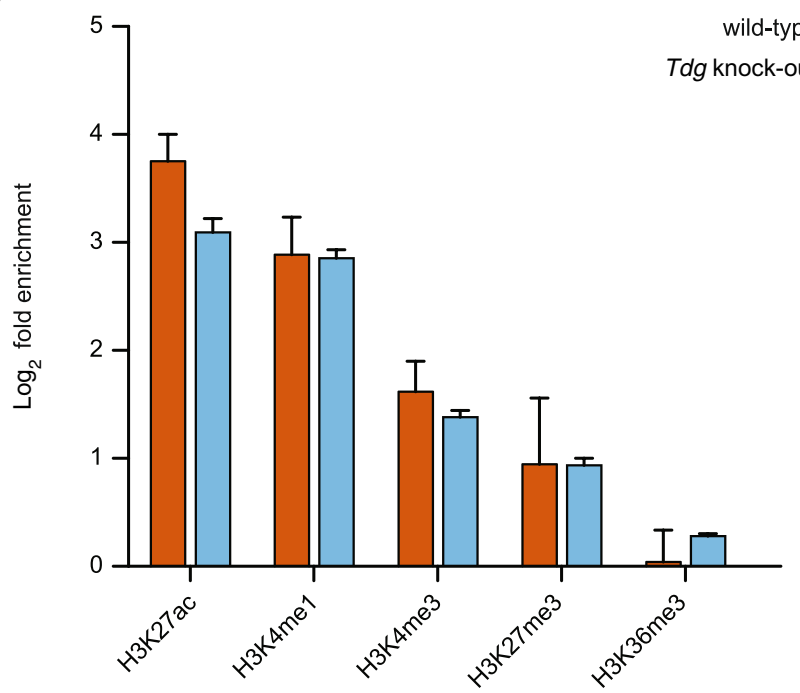

Fig. $35 f C$ is enriched at active enhancers in vivo. a Screenshot of a genomic region on chromosome 2 exemplifying the striking overlap of the $5 f C$ signal with regions marked by H3K4me1 (orange boxes) and H3K27ac (purple boxes), as calculated in [27]. b Fold enrichment of 5fC peaks in hindbrain and heart from WT and Tdg null embryos over regions marked by H3K4me1, H3K4me3 and H3K27ac in the brain and heart of E14.5 embryos, respectively. Log 2 fold enrichment was calculated for each replicate individually using 10,000 randomisations in a simulation procedure implemented with the GAT. Plotted is the average enrichment with standard deviation. c Comparison of $5 \mathrm{fC}$ peak enrichment in the hindbrain in regions marked by different histone modifications in the E14.5 mouse brain 


\section{Tissue-specific cytosine formylation at developmental enhancers}

We next investigated whether the strong enrichment detected at active enhancers was regulated in a tissuespecific fashion. To answer this question, we analysed $5 \mathrm{fC}$ enrichment in heart-specific, hindbrain-specific and liver-specific enhancers, obtained from the ENCODE database (Additional file 2: Table S1), in the various tissues. We found that in hindbrain tissues from both WT and $T d g$ null embryos, $5 \mathrm{fC}$ was considerably enriched at brain-specific enhancers, compared to heart-specific and liver-specific ones. Similarly, heart-specific enhancers were specifically enriched in the heart samples. (Fig. 4a). These findings strongly indicate not only that there is a dynamic regulation of $5 \mathrm{fC}$ at the level of enhancers, but also that this complex mechanism is specifically focused at tissue-specific developmental enhancers. In line with this observation, TET-mediated active demethylation of developmental enhancers has been recently shown to be a conserved mechanism in vertebrate embryogenesis [29].

Finally, we investigated the relationship between tissue-specific $5 \mathrm{fC}$ formation and gene function. To this end, we subdivided $5 \mathrm{fC}$ peaks in the individual tissues from $T d g$ null embryos into shared and tissue-specific peaks. Notably, heart-specific $5 \mathrm{fC}$ peaks were enriched in the proximity of genes involved in heart development, whereas hindbrain-specific peaks were enriched proximal to genes involved in neuron development (Fig. 4b and example in Additional file 1: Figure S8). Interestingly we could not find a similar enrichment for liver-specific $5 \mathrm{fC}$ peaks, which may be due to the mixed endoderm/mesoderm lineage of this tissue [30]. Taken together, these results clearly indicate the presence of a tissue-specific role for $5 \mathrm{fC}$ formation at the level of genes involved in the development of the cognate tissues.

\section{Conclusions}

We have generated the first in vivo genome-wide profile for $5 \mathrm{fC}$ in tissues obtained from WT and Tdg null E11.5 mouse embryos; namely from dissected heart, hindbrain and liver tissues and the carcass. While showing an overall similar profile to the ones reported in mESCs, with enrichment at enhancers and exons, we also find that $5 \mathrm{fC}$ is specifically enriched at active enhancers in the developing embryo. Moreover, we report clear tissue specificity behind the distribution of $5 \mathrm{fC}$ in developmentally related enhancers. This highlights a complex regulation of $5 \mathrm{fC}$, balancing of TET oxidation and TDG excision, and thus suggesting a key role for dynamic demethylation at those sites. The relationship between this process and the striking phenotype displayed by $T d g$-deficient embryos warrants future investigation.

\section{Methods}

\section{Knockout mouse and tissue dissection}

$T d g$ null mice were described in [21]. These mice contain a deletion of the coding sequence for the catalytic domain of TDG, which is replaced by a neomycin-resistant cassette. Genotyping was carried out using DNA isolated from the yolk sac as template with the following primers:

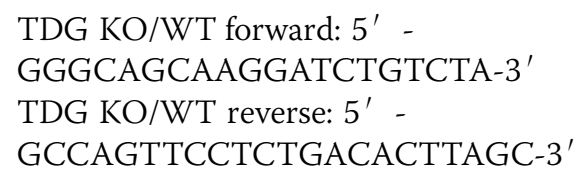

Mice were maintained as heterozygotes and intercrossed to generate homozygous knockout embryos for analysis. Tissues were collected from timed matings with the day of the copulation plug as E0.5. Embryos were dissected into hindbrain, heart, liver and carcass.

\section{DNA and RNA extraction}

Embryonic tissues were lysed in RLT plus buffer (Qiagen), homogenised via centrifugation through a QIAshreddred column (Qiagen) at full speed for 2 min in a microcentrifuge. DNA and RNA were isolated using AllPrep DNA/RNA kit (Qiagen) following the manufacturer's instructions.

\section{Mass spectrometry of DNA}

A total of 100-300 ng of genomic DNA was treated with $5 \mathrm{U}$ of DNA Degradase Plus (Zymo Research) for $3 \mathrm{~h}$ and analysed on a nanoHPLC combined with a Q Exactive mass spectrometer, as previously described [31].

\section{Pull-down method}

Genomic DNA (500 ng in $10 \mathrm{mM}$ Tris- $\mathrm{HCl} \mathrm{pH} 8$, $1 \mathrm{mM}$ EDTA) was sonicated to an average of $250 \mathrm{bp}$ with the Covaris M220 Focused-ultrasonicator. Synthetic spike-in controls were added after sonication to monitor the enrichment efficiency (full details on the spike-in oligos and conditions used are in Additional file 3). The fragmented DNA was incubated with a cleavable aldehyde reactive biotinylation probe [22] $(400 \mu \mathrm{M}), p$-anisidine $(100 \mathrm{mM})$ and $\mathrm{NH}_{4} \mathrm{OAc}(40 \mathrm{mM}$, adjusted to $\mathrm{pH} 5$ with $\mathrm{AcOH})$ in a final volume of $100 \mu \mathrm{L}$ at $37{ }^{\circ} \mathrm{C}$ with a $65{ }^{\circ} \mathrm{C}$ heated lid for $24 \mathrm{~h}$. The DNA was purified on silica-based spin columns (GeneJET PCR Purification Kit), with the inclusion of an additional wash step, before standard library preparation.

Streptavidin paramagnetic particles $(50 \mu \mathrm{L}$, Promega Magnesphere PMPs) were washed with $1 \times$ binding buffer (5 mM Tris- $\mathrm{HCl} \mathrm{pH} \mathrm{7.2,} 0.5 \mathrm{mM}$ EDTA, $1 \mathrm{M} \mathrm{NaCl}$, $0.05 \%$ Tween-20) (four times $500 \mu \mathrm{L}$ ) and resuspended in $2 \times$ binding buffer $(50 \mu \mathrm{L})$. The washed beads $(50 \mu \mathrm{L})$, dIdC $(2 \mu \mathrm{L}$ of $1 \mu \mathrm{g} / \mu \mathrm{L})$, spike-in control sequences 
A

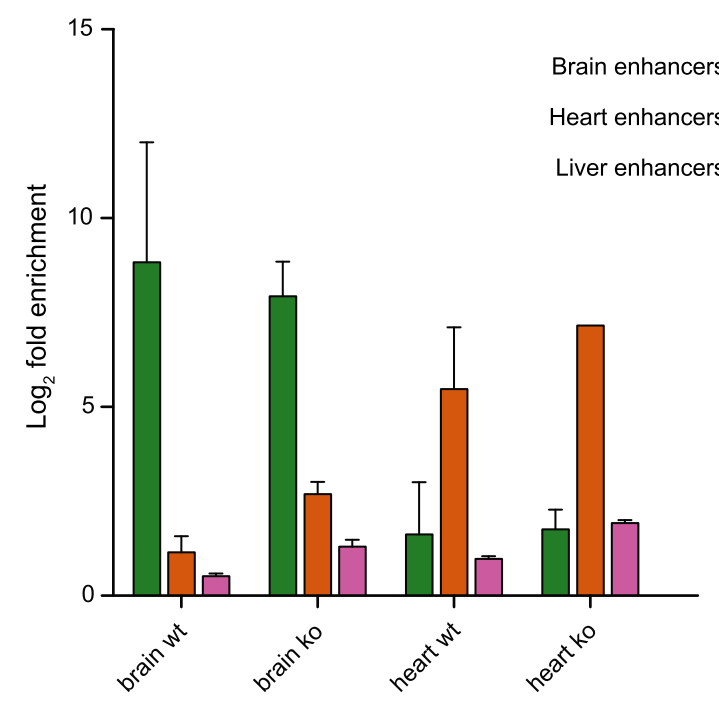

B
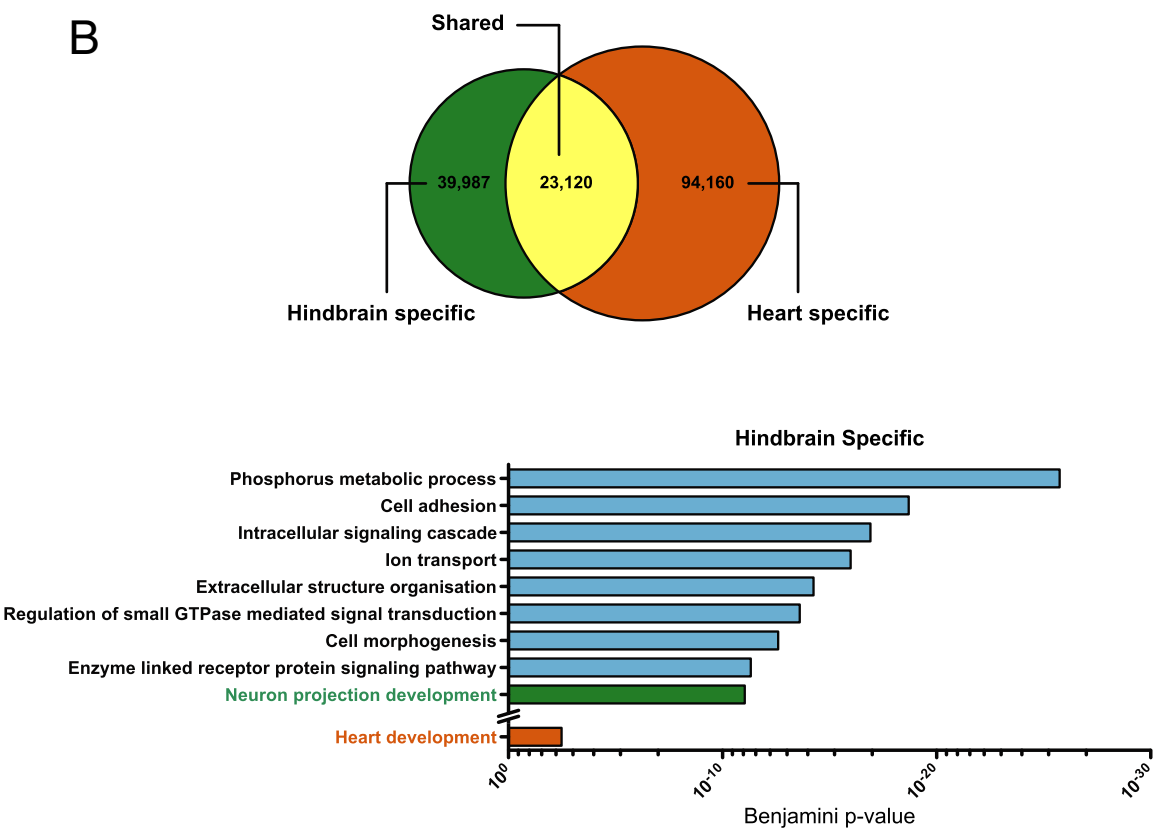

Heart Specific

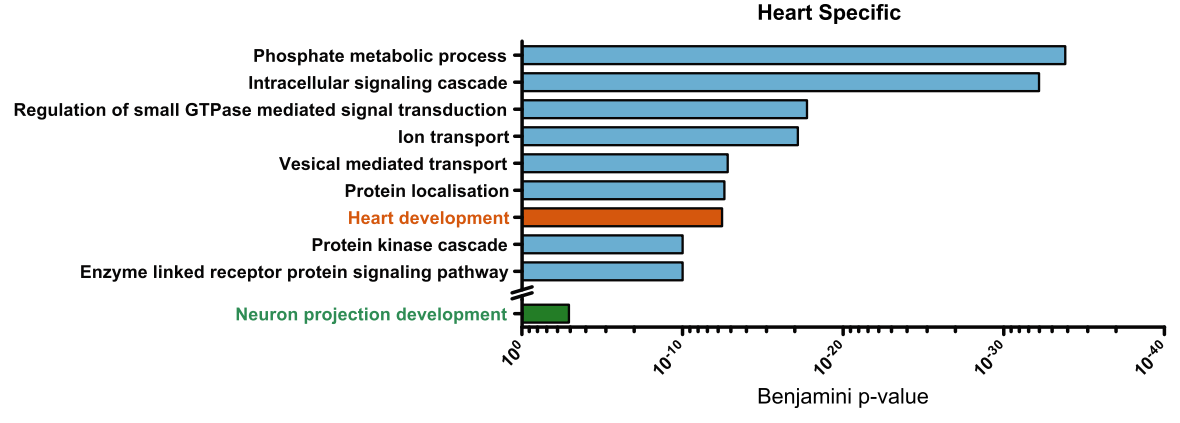

Fig. 4 (See legend on next page.) 
(See figure on previous page.)

Fig. 4 Tissue-specific 5fC formation at developmental enhancers. a Enrichment of $5 f C$ peaks in the different tissues over tissue-specific enhancer regions. $\log _{2}$ fold enrichment was calculated for each replicate individually using 10,000 randomisations in a simulation procedure implemented with the GAT. Plotted is the average enrichment with standard deviation. b Comparison of $5 \mathrm{fC}$ peaks detected specifically in the hindbrain and heart samples. Gene Ontology analysis was performed on genes in proximity (1 kb cutoff) of hindbrain-specific and heart-specific peaks

(1 pg positive control; $10 \mathrm{pg}$ negative control), DNA library and water were combined in a $1.5 \mathrm{~mL}$ low binding tube (Eppendorf DNA LoBind) to give $100 \mu \mathrm{L}$. The mixture was incubated at room temperature for $20 \mathrm{~min}$, before the tube was placed on a magnetic rack and left for 5 min while the magnetic beads formed a pellet. Once the solution was clear the supernatant was discarded, and $400 \mu \mathrm{L} 1 \times$ binding buffer was added to the tube. The beads were washed by rapidly rotating the tube $180^{\circ}$ and allowing the beads to recollect against the magnet; this washing procedure was repeated four times. The beads were resuspended in $400 \mu \mathrm{L} 1 \times$ binding buffer with vortexing and transferred to a new low binding tube before the washing procedure was repeated another four times. A final tube transfer and washing cycle was performed, giving a total of two tube changes and 12 washes. A final wash with Tris- $\mathrm{HCl}(10 \mathrm{mM})$ was performed, with the beads remaining on the magnet at all times due to poor pellet formation in this buffer.

The DNA was eluted from the beads by chemical cleavage of the probe with $100 \mu \mathrm{L}$ of tris(2-carboxyethyl)phosphine $(100 \mathrm{mM})$ and Tris- $\mathrm{HCl}(200 \mathrm{mM})$ shaken at $65{ }^{\circ} \mathrm{C}$ for $15 \mathrm{~min}$. NaOAc $(40 \mu \mathrm{L}$ of $5 \mathrm{M}$, $\mathrm{pH} 5)$, ethanol $(1 \mathrm{~mL})$ and water $(100 \mu \mathrm{L})$ were added to the isolated supernatant, and the mixture held at $-80{ }^{\circ} \mathrm{C}$ for $45 \mathrm{~min}$. The precipitated DNA was pelleted by centrifugation at 16,000 $\mathrm{rcf}$ for $45 \mathrm{~min}$, washed with $70 \%$ $\mathrm{EtOH}$ and centrifuged again at $16,000 \mathrm{rcf}$ for $30 \mathrm{~min}$. The pellet was air dried and dissolved in water $(20 \mu \mathrm{L})$. At this point the enrichment efficiency was examined by qPCR of the control sequences spiked in before pull-down. One microliter of enriched DNA was diluted tenfold, from which $1 \mu \mathrm{L}$ aliquots were amplified with primer sets for the positive and negative controls. Comparison to a non-enriched input gave the enrichment factor (Additional file 1: Figure S9 and Additional file 3). Although the enrichment was extremely high ( $\sim 44,000$-fold), there will be a proportion of false-positive regions arising from the excess of non-formylated DNA in genomic context.

\section{Library preparation and sequencing}

Sequencing libraries were generated using the NEBNext library preparation modules. Fourteen cycles of PCR amplification were performed with the Illumina PCR Master Mix and PCR Primer Cocktail. Prior to sequencing, libraries were quantified by qPCR (KAPA Biosystems) and their fragment size profile analysed by TapeStation (Agilent).
Sequencing was performed on an Illumina Nextseq 500, run in paired-end mode with 100 read cycles.

\section{Bioinformatic analysis}

Raw sequencing files and peak files can be found in the GEO under accession number GSE77447. Data were adapter and quality trimmed using trim_galore v0.4.1 (bioinformatics.babraham.ac.uk/projects/trim_galore/) using default settings. Trimmed sequencing reads were aligned to mouse genome assembly NCBIM37 (mm9) using Bowtie2 v2.2.6 using default parameters [32] and was converted to BAM format using samtools v0.1.18 [33]. Peaks were called in the SeqMonk software (bioinformatics.babraham.ac.uk/projects/seqmonk/) using the MACS peak caller [34], with a $p$ value cutoff of $1 \times 10^{-5}$ and a fragment size of $200 \mathrm{bp}$. Data manipulations were facilitated by BEDTools [35] and data analysis was performed in Seqmonk or in R version 3.1.2 [36].

\section{Gene Ontology}

Functional annotation enrichment analyses were performed using the Database for Annotation, Visualization and Integrated Discovery (DAVID) v6.7 [37].

\section{Additional files}

Additional file 1: Supplementary figures and supplementary figure legends. (PDF $841 \mathrm{~kb}$ )

Additional file 2: Table S1. ENCODE datasets analysed. (PDF $53 \mathrm{~kb}$ ) Additional file 3: Full details on spike-in control oligos. (PDF 226 kb)

\section{Abbreviations}

5caC, 5-carboxylcytosine; 5fC, 5-formylcytosine; 5hmC, 5-hydroxymethylcytosine: 5mC, 5-methylcytosine; BER, base excision repair; BS-seq, bisulphite sequencing; CGl, CpG island; GAT, genome association tester; LC/MS, liquid chromatography/ mass spectrometry; mESC, mouse embryonic stem cells; NPC, neural precursor cells; TDG, thymine DNA glycosylase; WT, wild type

\section{Acknowledgements}

We would like to thank Simon Andrews for his bioinformatics support, Primo Schär for the Tdg KO mice and David Oxley and Judith Webster for their help with mass spectrometry.

\section{Funding}

$\mathrm{Ml}$ is supported by the People Programme (Marie Curie Actions) of the European Union's Seventh Framework Programme FP7/2007-2013/under REA grant agreement no. 290123. GRM was supported by Trinity College and Herchel Smith studentships. MB was supported by the CRUK PhD Training Programme in Chemical Biology and Molecular Medicine. DB is supported by funding from the Wellcome Trust and Herchel Smith. The WR lab is supported by BBSRC, MRC, the Wellcome Trust, EU EpiGeneSys and BLUEPRINT. The SB lab is supported by core funding from Cancer Research UK and a Wellcome Trust Senior Investigator Award. 


\section{Availability of data and materials}

The data discussed in this publication are accessible through GEO Series accession number GSE77447 (https://www.ncbi.nlm.nih.gov/geo/query/ acc.cgi?acc=GSE77447).

\section{Authors' contributions}

$\mathrm{MI}$ and GRM conceived the study, performed the experiments, analysed the data and wrote the manuscript. $\mathrm{HB}$ and $\mathrm{MB}$ generated mass spectrometry data. WD performed tissue dissections. EAR and HB conceived the study and analysed the data. DB performed bioinformatics analysis. WD, SB and WR conceived the study and provided supervision. All authors have interpreted the data, read and approved the manuscript.

\section{Competing interests}

SB and WR are advisors and shareholders of Cambridge Epigenetix, Ltd.

\section{Ethics approval and consent to participate}

All experimental procedures were approved by the Animal Welfare, Experimentation and Ethics Committee at the Babraham Institute and were performed under licenses by the Home Office (UK) in accordance with the Animals (Scientific Procedures) Act 1986.

\section{Author details}

${ }^{1}$ The Babraham Institute, Epigenetics Programme, Cambridge CB22 3AT, UK. ${ }^{2}$ Department of Chemistry, University of Cambridge, Lensfield Road, Cambridge CB2 1EW, UK. ${ }^{3}$ Cancer Research UK, Cambridge Institute, Li Ka Shing Centre, Cambridge CB2 ORE, UK. ${ }^{4}$ Present Address: Discovery Sciences, AstraZeneca, Alderley Park, Macclesfield SK10 4TG, UK. ${ }^{5}$ School of Clinical Medicine, University of Cambridge, Cambridge CB2 OSP, UK. ${ }^{6}$ Centre for Trophoblast Research, University of Cambridge, Cambridge CB2 3EG, UK. ${ }^{7}$ Wellcome Trust Sanger Institute, Hinxton CB10 1SA, UK.

\section{Received: 4 March 2016 Accepted: 6 June 2016}

\section{Published online: 29 June 2016}

\section{References}

1. Tahiliani M, Koh KP, Shen Y, Pastor WA, Bandukwala H, Brudno Y, et al. Conversion of 5-methylcytosine to 5-hydroxymethylcytosine in mammalian DNA by MLL partner TET1. Science. 2009;324:930-5.

2. Ito S, D'Alessio AC, Taranova OV, Hong K, Sowers LC, Zhang Y. Role of Tet proteins in $5 \mathrm{mC}$ to $5 \mathrm{hmC}$ conversion, ES-cell self-renewal and inner cell mass specification. Nature. 2010;466:1129-33.

3. Kriaucionis $\mathrm{S}$, Heintz N. The nuclear DNA base 5-hydroxymethylcytosine is present in Purkinje neurons and the brain. Science. 2009;324:929-30.

4. Ito S, Shen L, Dai Q, Wu SC, Collins LB, Swenberg JA, et al. Tet proteins can convert 5-methylcytosine to 5-formylcytosine and 5-carboxylcytosine. Science. 2011:333:1300-3.

5. He YF, Li BZ, Li Z, Liu P, Wang Y, Tang Q, et al. Tet-mediated formation of 5-carboxylcytosine and its excision by TDG in mammalian DNA. Science. 2011;333:1303-7

6. Maiti A, Drohat AC. Thymine DNA glycosylase can rapidly excise 5formylcytosine and 5-carboxylcytosine: potential implications for active demethylation of CpG sites. J Biol Chem. 2011;286:35334-8.

7. Pfaffeneder T, Hackner B, Truss M, Münzel M, Müller M, Deiml CA, et al. The discovery of 5-formylcytosine in embryonic stem cell DNA. Angew Chem Int Ed Engl. 2011;50:7008-12.

8. Bachman M, Uribe-Lewis S, Yang X, Burgess HE, lurlaro M, Reik W, et al. 5-Formylcytosine can be a stable DNA modification in mammals. Nat Chem Biol. 2015;11:555-7.

9. Iurlaro M, Ficz G, Oxley D, Raiber E-A, Bachman M, Booth MJ, et al. A screen for hydroxymethylcytosine and formylcytosine binding proteins suggests functions in transcription and chromatin regulation. Genome Biol. 2013;14:R119.

10. Spruijt CG, Gnerlich F, Smits AH, Pfaffeneder T, Jansen PWTC, Bauer C, et al. Dynamic readers for 5-(hydroxy)methylcytosine and its oxidized derivatives. Cell. 2013:152:1146-59.

11. Raiber E-A, Murat P, Chirgadze DY, Beraldi D, Luisi BF, Balasubramanian S. 5Formylcytosine alters the structure of the DNA double helix. Nat Struct Mol Biol. 2015;22:44-9.

12. Raiber EA, Beraldi D, Ficz G, Burgess HE, Branco MR, Murat P, et al. Genomewide distribution of 5-formylcytosine in embryonic stem cells is associated with transcription and depends on thymine DNA glycosylase. Genome Biol. 2012;13:R69.

13. Song C-X, Szulwach KE, Dai Q, Fu Y, Mao S-Q, Lin L, et al. Genome-wide profiling of 5 -formylcytosine reveals its roles in epigenetic priming. Cell. 2013;153:678-91.

14. Shen L, Wu H, Diep D, Yamaguchi S, D'Alessio AC, Fung H-L, et al. Genomewide analysis reveals TET- and TDG-dependent 5-methylcytosine oxidation dynamics. Cell. 2013;153:692-706.

15. Booth MJ, Marsico G, Bachman M, Beraldi D, Balasubramanian S. Quantitative sequencing of 5-formylcytosine in DNA at single-base resolution. Nat Chem. 2014:6:435-40.

16. Neri F, Incarnato D, Krepelova A, Rapelli S, Anselmi F, Parlato C, et al. Singlebase resolution analysis of 5-formyl and 5-carboxyl cytosine reveals promoter DNA methylation dynamics. Cell Rep. 2015;10:674-83.

17. Wu H, Wu X, Shen $L$, Zhang $Y$. Single-base resolution analysis of active DNA demethylation using methylase-assisted bisulfite sequencing. Nat Biotechnol. 2014;32:1231-40.

18. Xia B, Han D, Lu X, Sun Z, Zhou A, Yin Q, et al. Bisulfite-free, base-resolution analysis of 5-formylcytosine at the genome scale. Nat Methods. 2015;12:1047-50.

19. Sun Z, Dai N, Borgaro JG, Quimby A, Sun D, Corrêa IR, et al. A sensitive approach to map genome-wide 5-hydroxymethylcytosine and 5formylcytosine at single-base resolution. Mol Cell. 2015:57:750-61.

20. Cortellino S, Xu J, Sannai M, Moore R, Caretti E, Cigliano A, et al. Thymine DNA glycosylase is essential for active DNA demethylation by linked deamination-base excision repair. Cell. 2011;146:67-79.

21. Cortázar D, Kunz C, Selfridge J, Lettieri T, Saito Y, MacDougall E, et al. Embryonic lethal phenotype reveals a function of TDG in maintaining epigenetic stability. Nature. 2011;470:419-23.

22. McInroy GR, Raiber E-A, Balasubramanian S. Chemical biology of genomic DNA: minimizing PCR bias. Chem Commun (Camb). 2014;50:12047-9.

23. Heger A, Webber C, Goodson M, Ponting CP, Lunter G. GAT: a simulation framework for testing the association of genomic intervals. Bioinformatics. 2013:29:2046-8

24. Weber $A R$, Krawczyk C, Robertson AB, Kuśnierczyk A, Vågbø CB, Schuermann $D$, et al. Biochemical reconstitution of TET1-TDG-BER-dependent active DNA demethylation reveals a highly coordinated mechanism. Nat Commun. 2016;7:10806.

25. Wen L, Li X, Yan L, Tan Y, Li R, Zhao Y, et al. Whole-genome analysis of 5hydroxymethylcytosine and 5-methylcytosine at base resolution in the human brain. Genome Biol. 2014;15:R49.

26. Kellinger MW, Song C-X, Chong J, Lu X-Y, He C, Wang D. 5-formylcytosine and 5-carboxylcytosine reduce the rate and substrate specificity of RNA polymerase II transcription. Nat Struct Mol Biol. 2012;19:831-3.

27. Mouse ENCODE. Consortium, Stamatoyannopoulos JA, Snyder M, Hardison R, Ren B, Gingeras T, et al. An encyclopedia of mouse DNA elements (Mouse ENCODE). Genome Biol. 2012;13:418.

28. Neri F, Incarnato D, Krepelova A, Rapelli S, Pagnani A, Zecchina R, et al. Genome-wide analysis identifies a functional association of Tet1 and Polycomb repressive complex 2 in mouse embryonic stem cells. Genome Biol. 2013;14:R91.

29. Bogdanović O, Smits AH, de la Calle ME, Tena JJ, Ford E, Williams R, et al. Active DNA demethylation at enhancers during the vertebrate phylotypic period. Nat Genet. 2016;48:417-26.

30. Zhao R, Duncan SA. Embryonic development of the liver. Hepatology. 2005 41:956-67.

31. Bachman M, Uribe-Lewis S, Yang X, Williams M, Murrell A, Balasubramanian S 5-Hydroxymethylcytosine is a predominantly stable DNA modification. Nat Chem. 2014;6:1049-55

32. Langmead B, Salzberg SL. Fast gapped-read alignment with Bowtie 2. Nat Methods. 2012;9:357-9.

33. Li H, Handsaker B, Wysoker A, Fennell T, Ruan J, Homer N, et al. The Sequence Alignment/Map format and SAMtools. Bioinformatics. 2009;25:2078-9.

34. Zhang $Y$, Liu T, Meyer CA, Eeckhoute J, Johnson DS, Bernstein BE, et al. Model-based analysis of ChIP-Seq (MACS). Genome Biol. 2008;9:R137.

35. Quinlan AR, Hall IM. BEDTools: a flexible suite of utilities for comparing genomic features. Bioinformatics. 2010;26:841-2.

36. Team RDC. R: A Language and Environment for Statistical Computing Vienna: R Foundation; 2012.

37. Huang DW, Sherman BT, Lempicki RA. Systematic and integrative analysis of large gene lists using DAVID bioinformatics resources. Nat Protoc. 2009;4: 44-57. 